\title{
RINGS HAVING DOMINANT MODULES
}

\author{
TOYONORI KATO
}

(Received April 19, 1971)

Recently the notion of dominant modules has been introduced in Kato [9] prompted by Tachikawa [17] and then studied further in Kato [10]. In this paper we shall be concerned with a class of rings which includes the class of left perfect rings as well as the class of left $S$-rings, namely, rings having dominant left modules.

Section 1 is devoted to illustrative examples of such rings, most of which are quoted from [9].

On the other hand, there appeared in Morita [13, 15] (cf. Jans [5]) the following condition on a ring $R$

$$
\left.\operatorname{Hom}\left(\operatorname{Ext}^{1}{ }_{R} X,{ }_{R} R\right)_{R}, E\left(R_{R}\right)\right)=0
$$

for (finitely generated) ${ }_{R} X \in_{R} \mathscr{C l}$, where and throughout this paper, $E(\quad$ ) will denote the injective hull, and ${ }_{R} \mathscr{C}$ the category of left $R$-modules.

For the class of rings having dominant left modules, this condition (2) characterizes left QF-3 rings $\mathrm{s}^{1)}$; the proof of this theorem is given in Section 2. The point of this theorem is that the converse of Morita [13, Theorem 4.1] holds.

It was Lambek [11] who pointed out for the first time that Utumi's maximal right quotient ring of a ring $R$ (cf. Utumi [19]) is the bicommutator of $E\left(R_{R}\right)$. In what follows, let $Q$ be Utumi-Lambek maximal right quotient ring of a ring $R$. If $R$ has a dominant left module, so does $Q$ (Example 8 in Section 3). This observation leads us to investigate the situation when $Q$ has a dominant left module. The purpose of Section 3, the final section, is to examine this situation entirely based on Morita [14]. It is shown in Theorem 2 that $Q$ has a dominant left module if and only if there exists a module ${ }_{R} U$ such that

(i) ${ }_{R} U$ is of type $F P$.

(ii) ${ }_{R} U$ is faithful and flat.

(iii) $U_{S}$ is lower distinguished, where $S=\operatorname{End}\left({ }_{R} U\right)$.

For an illustrative example of this situation, let $R=Z$ be the ring of integers and ${ }_{R} U={ }_{z} Q$ the rational number field. In this connection, if

1) A ring $R$ is called left $\mathrm{QF}-3$ if $E\left({ }_{R} R\right)$ is torsionless (cf. Colby and Rutter [4], Tachikawa [17] and Kato [6, 7]). 
${ }_{Q} U$ is dominant, then

$$
\operatorname{Hom}\left({ }_{R} Y,{ }_{R} Q\right) \otimes{ }_{R} U \approx \operatorname{Hom}\left({ }_{R} Y,{ }_{R} U\right)
$$

canonically for ${ }_{R} Y \in \in_{R} \mathscr{C l}$, and

$$
\operatorname{Hom}\left({ }_{R} Y,{ }_{R} R\right) \otimes{ }_{R} U \approx \operatorname{Hom}\left({ }_{R} Y,{ }_{R} U\right)
$$

canonically for finitely generated ${ }_{R} Y \in_{R} \mathscr{L}$, as is shown in Lemma 4. Theorem 3 discusses the situation when ${ }_{R} U$ is injective for a dominant module ${ }_{Q} U$. Among other things it is shown that, if there exists a dominant module ${ }_{Q} U$ such that ${ }_{R} U$ is injective, then the condition (2) above holds for all finitely generated modules ${ }_{R} X$. Theorem 3 contains the converse part of Morita [15, Theorem 2] for the class of left Noetherian rings $R$ for which $Q$ has dominant left modules as well.

Throughout this paper, rings $R$ will have unity element and modules will be unital. ${ }_{R} X$ will signify the fact that $X$ is a left $R$-module. As a matter of course, homomorphisms of modules will operate on the side opposite to the scalars.

1. Introduction to dominant modules. A faithful, finitely generated, projective module ${ }_{R} U$ is called dominant if $U_{S}$ is lower distinguished ${ }^{2}$, where $S=\operatorname{End}\left({ }_{R} U\right)$ is the endomorphism ring of ${ }_{R} U$ (cf. Kato [9]). In this paper we are mainly concerned with rings having dominant modules, and so let us survey such rings by illustrative examples:

EXAMPLE 1. A progenerator ${ }_{R} U^{3)}$ is dominant if and only if $R_{R}$ is lower distinguished.

This follows from the Morita equivalence $\mathscr{M}_{S} \sim \mathscr{M}_{R}, S=\operatorname{End}\left({ }_{R} U\right)$.

The following example is an analogue of [9, Example 3] (cf. Morita [14, Theorem 8.2]).

EXAMPLE 2. $R$ has a dominant left module and $E\left(R_{R}\right)$-domi. $\operatorname{dim} R_{R} \geqq 2^{4)}$ if and only if $R$ is the endomorphism ring of a lower distinguished generator for $\mathscr{M}_{S}$, where $S$ is a ring.

ExAMPle 3 (Kato [9, Example 4]). If $R$ is a semi-perfect ring with the essential right socle, then $R$ has a dominant left module. Thus left perfect rings as well as semi-primary rings have always dominant left modules.

EXAMPLE 4. The ring $Z$ of integers has no dominant module.

\footnotetext{
2) $U_{S}$ contains a copy of each simple right $S$-module (cf. Azumaya [1]).

3) ${ }_{R} U$ is a finitely generated projective generator for ${ }_{R} \mathscr{M}$ (cf. Bass [2]).

4) $E\left(R_{R}\right) / R G \Pi E\left(R_{R}\right)$ (cf. Tachikawa [17, 18], Morita [14] and Kato [8]).
} 
Azumaya's observation [1, Theorem 8] and Example 1 above will serve a verification of this example.

Example 5. Let $R$ be an infinite direct product of fields. Then $R$ has no dominant module, and yet $R$ is a commutative, self-injective, regular ring (cf. [9, Example 2]).

2. Characterization of QF-3 rings. In this section we are chiefly concerned with rings $R$ having dominant left modules, and then give a characterization of left QF-3 rings in terms of the condition (2) mentioned in Introduction.

Lemma 1. Let ${ }_{R} U$ be a dominant module. Then $E\left({ }_{R} R\right)$ is torsionless if and only if ${ }_{R} U$ is injective.

PRoof. The "if" part follows directly from Kato [6, Proposition 1]. To show the "only if" part, suppose $E\left({ }_{R} R\right)$ is torsionless. We observe first that $E\left({ }_{R} U\right)$ is $U$-torsionless. Indeed, since ${ }_{R} U \subseteq \Pi_{R} R \subseteq \Pi E\left({ }_{R} R\right)$, $E\left({ }_{R} R\right) \subseteq \Pi_{R} R$, and ${ }_{R} R \subseteq \Pi_{R} U$ by assumption,

$$
E\left({ }_{R} U\right) G \Pi E\left({ }_{R} R\right) G \Pi_{R} R G \Pi_{R} U .
$$

Observe next that $U_{S}$ is lower distinguished, where $S=\operatorname{End}\left({ }_{R} U\right)$. Thus, according to Onodera [16, Lemma 4.4$]^{5},{ }_{R} U$ is injective.

Lemma 2 (Kato [9]). Let ${ }_{R} U$ be faithful, finitely generated projective and $S=\operatorname{End}\left({ }_{R} U\right)$. Then

$$
\operatorname{Hom}\left(U_{S}, E\left(U_{S}\right)\right)_{R}=E\left(R_{R}\right)^{6)} \text {. }
$$

Lemma 3 (Morita [15, Theorem 2'] $)^{7}$. If $R$ has a faithful, finitely generated projective, injective left module, then

$$
\operatorname{Hom}\left(\operatorname{Ext}^{1}\left({ }_{R} X,{ }_{R} R\right)_{R}, E\left(R_{R}\right)\right)=0 \text { for }{ }_{R} X \in_{R} \mathscr{K C} \text {. }
$$

REMARK. If $R$ has a faithful, projective, injective left module, then

$$
\left.\operatorname{Hom}\left(\operatorname{Ext}^{1}{ }_{R} X,{ }_{R} R\right)_{R}, E\left(R_{R}\right)\right)=0
$$

for finitely generated ${ }_{R} X \in_{R} \mathscr{C}$.

We shall sketch the proof. Given ${ }_{R} U$ and ${ }_{R} Y$, there exists the canonical map

$$
\alpha: \operatorname{Hom}\left({ }_{R} Y,{ }_{R} R\right) \otimes{ }_{R} U \longrightarrow \operatorname{Hom}\left({ }_{R} Y,{ }_{R} U\right)
$$

\footnotetext{
5) By a slight modification of the proof of [6, Lemma 1], the author obtained this result independently.

6) The author is grateful to Dr. T. Onodera who showed him another simple proof (cf. forthcoming papers T. Onodera [Eine Bemerkung uber Kogeneratoren] and T. Kato [ $U$-distinguished modules]).

7) This has also been independently obtained by the author.
} 
defined via

$$
y((f \otimes u) \alpha)=(y f) u \quad \text { for } y \in Y, f \in \operatorname{Hom}\left({ }_{R} Y,{ }_{R} R\right), u \in U \text {. }
$$

It is known that $\alpha$ is a monomorphism for ${ }_{R} Y \in \in_{R} \mathscr{A}$, if ${ }_{R} U$ is projective. With this fact in mind, assume now that ${ }_{R} U$ is faithful, projective, and injective. Then an exact sequence $0 \rightarrow{ }_{R} Y \rightarrow{ }_{R} P \rightarrow{ }_{R} X \rightarrow 0$ with ${ }_{R} P$ finitely generated projective, gives rise to the following commutative diagram with exact rows

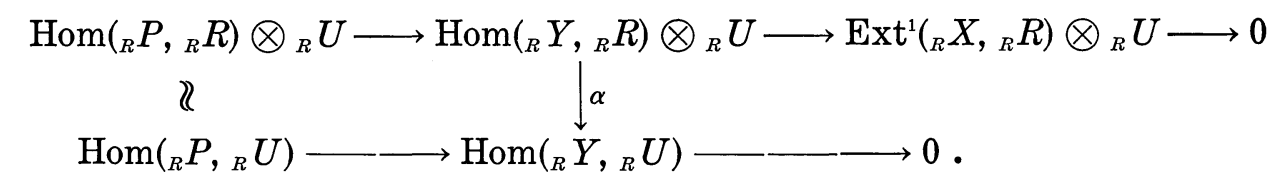

Hence $\operatorname{Ext}^{1}\left({ }_{R} X,{ }_{R} R\right) \otimes{ }_{R} U=0$ since $\alpha$ is a monomorphism. On the other hand, since ${ }_{R} U$ is faithful and projective,

$$
E\left(R_{R}\right) G \operatorname{Hom}\left(U_{S}, E\left(U_{S}\right)\right)_{R} ; \quad S=\operatorname{End}\left({ }_{R} U\right) .
$$

It thus follows

$$
\begin{gathered}
\operatorname{Hom}\left(\operatorname{Ext}^{1}\left({ }_{R} X,{ }_{R} R\right)_{R}, E\left(R_{R}\right)\right) \subset \operatorname{Hom}\left(\operatorname{Ext}^{1}\left({ }_{R} X,{ }_{R} R\right)_{R}, \operatorname{Hom}\left(U_{S}, E\left(U_{S}\right)\right)_{R}\right) \\
\approx \operatorname{Hom}\left(\operatorname{Ext}^{1}\left({ }_{R} X,{ }_{R} R\right) \otimes{ }_{R} U_{S}, E\left(U_{S}\right)\right)=0 .
\end{gathered}
$$

We are now ready for our main theorem.

THEOREM 1. If $R$ has a dominant left module, then the following conditions are equivalent:

(1) $E\left({ }_{R} R\right)$ is torsionless.

(2) $\operatorname{Hom}\left(\operatorname{Ext}^{1}\left({ }_{R} X,{ }_{R} R\right)_{R}, E\left(R_{R}\right)\right)=0$ for ${ }_{R} X \in{ }_{R} \mathscr{M}$.

(2') $\left.\operatorname{Hom}\left(\operatorname{Ext}^{1}{ }_{R} R,{ }_{R} R\right)_{R}, E\left(R_{R}\right)\right)=0$

for finitely generated ${ }_{R} X \in_{R} \mathscr{M}$.

Proof. $\quad(1) \Longrightarrow(2)$. Let ${ }_{R} U$ be a dominant module. Since $E\left({ }_{R} R\right)$ is torsionless, ${ }_{R} U$ is injective by Lemma 1 . Now, ${ }_{R} U$ is faithful, finitely generated projective, and injective. Thus the condition (2) follows at once from Lemma 3.

$(2) \Rightarrow\left(2^{\prime}\right)$ is trivial.

$\left(2^{\prime}\right) \Longrightarrow(1)$. It suffices to show that ${ }_{R} U$ is injective, where ${ }_{R} U$ is dominant, in view of Lemma 1 . Let $0 \rightarrow{ }_{R} Y \rightarrow{ }_{R} P \rightarrow{ }_{R} X \rightarrow 0$ be an exact sequence with ${ }_{R} P$ finitely generated projective. In the same manner as above, we have the following exact commutative diagram

$$
\begin{gathered}
\left.\operatorname{Hom}\left({ }_{R} P,{ }_{R} R\right) \otimes{ }_{R} U \longrightarrow \operatorname{Hom}_{R} Y,{ }_{R} R\right) \otimes{ }_{R} U \longrightarrow \operatorname{Ext}^{1}\left({ }_{R} X,{ }_{R} R\right) \otimes_{R} U \longrightarrow 0 \\
\qquad \alpha \\
\operatorname{Hom}\left({ }_{R} P,{ }_{R} U\right) \longrightarrow \operatorname{Hom}\left({ }_{R} Y,{ }_{R} U\right),
\end{gathered}
$$


where the vertical maps $\alpha$ are isomorphisms by the finitely generated projectivity of ${ }_{R} U$ (cf. Morita [12, Lemma 7.1]). Here

$$
\operatorname{Ext}^{1}\left({ }_{R} X,{ }_{R} R\right) \otimes{ }_{R} U=0 \text {. }
$$

In fact,

$$
\begin{gathered}
\operatorname{Hom}\left(\operatorname{Ext}^{1}\left({ }_{R} X,{ }_{R} R\right) \otimes{ }_{R} U_{S}, E\left(U_{S}\right)\right) \approx \operatorname{Hom}\left(\operatorname{Ext}^{1}\left({ }_{R} X,{ }_{R} R\right)_{R}, \operatorname{Hom}\left(U_{S}, E\left(U_{S}\right)\right)_{R}\right) \\
\approx \operatorname{Hom}\left(\operatorname{Ext}^{1}\left({ }_{R} X,{ }_{R} R\right)_{R}, E\left(R_{R}\right)\right)=0 ; \quad S=\operatorname{End}\left({ }_{R} U\right)
\end{gathered}
$$

making use of Lemma 2 and the condition $\left(2^{\prime}\right)$. However $E\left(U_{S}\right)$ is a cogenerator for $\mathscr{C}_{S}$ since ${ }_{R} U$ is dominant. Therefore $\operatorname{Ext}^{1}\left({ }_{R} X,{ }_{R} R\right) \otimes_{R} U=0$. It now follows from the above diagram that the induced map $\operatorname{Hom}\left({ }_{R} P,{ }_{R} U\right) \rightarrow$ $\operatorname{Hom}\left({ }_{R} Y,{ }_{R} U\right)$ is an epimorphism. We have thus established the injectivity of ${ }_{R} U$.

REMARK. As we mentioned in Introduction, Theorem 1 is an improvement on Morita [13, Theorem 4.1], in view of Example 3 in Section 1.

The following two examples show that the "dominant" hypothesis is important in Theorem 1.

Example 6. According to Morita [15, Theorem 2] (cf. Theorem 3), the ring $Z$ of integers satisfies the condition $\left(2^{\prime}\right)$ above, whereas $E\left({ }_{z} Z\right)$ is not torsionless.

EXAmple $7^{8)}$. As is stated just above, the $\operatorname{ring} Z$ fulfils the condition $\left(2^{\prime}\right)$, but not the condition (2). In fact, let

$$
{ }_{Z} X=\bigoplus_{n=2}^{\infty} Z / n Z
$$

Then one verifies easily that

$$
\operatorname{Ext}^{1}\left({ }_{z} X,{ }_{z} Z\right) \approx \prod_{n=2}^{\infty} \operatorname{Ext}^{1}\left(Z / n Z,{ }_{z} Z\right) \approx \prod_{n=2}^{\infty} Z / n Z
$$

Thus

$$
\operatorname{Hom}\left(\operatorname{Ext}^{1}\left({ }_{z} X,{ }_{Z} Z\right)_{Z}, E\left(Z_{Z}\right)\right)=\operatorname{Hom}\left(\prod_{n=2}^{\infty} Z / n Z, Q_{Z}\right) \neq 0,
$$

where $Q$ is the rational number field.

3. Dominant modules over maximal quotient rings. In what follows, let $R$ be a ring and $Q$ Utumi-Lambek maximal right quotient ring of $R$ (cf. Lambek [11]). In this section we deal with rings $R$ for which $Q$ has a dominant left module.

ExAmple 8. If $R$ has a dominant left module, so does $Q$.

8) The author is indebted to Dr. K. Uchida for this example. 
Indeed, let ${ }_{R} U$ be dominant and $S=\operatorname{End}\left({ }_{R} U\right)$. Then $Q=\operatorname{End}\left(U_{S}\right)$ is Utumi-Lambek maximal right quotient ring of $R$ by Kato [10, Corollary 5]. Thus ${ }_{Q} U$ is dominant since $U_{S}$ is a lower distinguished generator for $\mathscr{N}_{S}$ (cf. Example 2).

The following theorem is entirely based on Morita [14].

THeorem 2. Let $R$ be a ring and $Q$ Utumi-Lambek maximal right quotient ring of $R$. Then the following conditions are equivalent:

(1) $Q$ has a dominant left module.

(2) There exists a module ${ }_{R} U$ such that

(i) ${ }_{R} U$ is of type $F P^{9)}$,

(ii) ${ }_{R} U$ is faithful and flat,

(iii) $U_{S}$ is lower distinguished, where $\left.S=\operatorname{End}_{R} U\right)$.

Proof. $(1) \Rightarrow(2)$. Let ${ }_{Q} U$ be dominant and $S=\operatorname{End}\left({ }_{Q} U\right)$. We shall now show that ${ }_{R} U$ satisfies (i), (ii), and (iii). By Lemma 2 and Lambek [11]

$$
\operatorname{Hom}\left(U_{S}, E\left(U_{S}\right)\right)_{Q}=E\left(Q_{Q}\right)=E\left(R_{R}\right) \text {. }
$$

Hence ${ }_{R} U$ is flat by Morita [14, Lemma 1.3], since $E\left(U_{S}\right)$ is an injective cogenerator for $\mathscr{M}_{s}$. On the other hand, since $Q$ is Utumi-Lambek maximal right quotient ring of $R$,

$$
\begin{gathered}
\operatorname{Hom}\left(Q / R \otimes{ }_{R} U_{S}, E\left(U_{S}\right)\right) \approx \operatorname{Hom}\left(Q / R, \operatorname{Hom}\left(U_{S}, E\left(U_{S}\right)\right)_{R}\right) \\
\approx \operatorname{Hom}\left(Q / R, E\left(R_{R}\right)\right)=0 .
\end{gathered}
$$

It follows that $Q / R \otimes{ }_{R} U=0$. Since ${ }_{R} U$ is flat, the exact sequence $0 \rightarrow$ $R_{R} \rightarrow Q_{R} \rightarrow Q / R \rightarrow 0$ induces an exact sequence

$$
0 \longrightarrow R \otimes_{R} U \longrightarrow Q \otimes_{R} U \longrightarrow Q / R \otimes_{R} U=0 \text {. }
$$

Thus

$$
{ }_{Q} U_{S} \approx{ }_{Q} Q \otimes{ }_{R} U_{S} \cdot
$$

Furthermore $U_{S}$ is a generator for $\mathscr{M}_{S}$ and $Q=\operatorname{End}\left(U_{S}\right)$. Thus, applying Morita [14, Theorem 1,1] we conclude that ${ }_{R} U$ is of type $F P$ and $S=\operatorname{End}\left({ }_{R} U\right)$.

$(2) \Rightarrow(1)$. Suppose ${ }_{R} U$ satisfies (i), (ii), and (iii). Let $S=\operatorname{End}\left({ }_{R} U\right)$ and $R^{\prime}=\operatorname{End}\left(U_{S}\right)$. From the flatness of ${ }_{R} U$, it follows that

$$
E\left(R_{R}^{\prime}\right) G \operatorname{Hom}\left(U_{S}, E\left(U_{S}\right)\right)_{R},
$$

and hence

$$
\begin{gathered}
\operatorname{Hom}\left(R^{\prime} / R, E\left(R_{R}^{\prime}\right)\right) G \operatorname{Hom}\left(R^{\prime} / R, \operatorname{Hom}\left(U_{S}, E\left(U_{S}\right)\right)_{R}\right) \\
\approx \operatorname{Hom}\left(R^{\prime} / R \otimes_{R} U_{S}, E\left(U_{S}\right)\right)=0,
\end{gathered}
$$

\footnotetext{
9) For the definition, see Morita $[14, \S 1]$.
} 
for, ${ }_{R} U$ is of type $F P$. This implies that $R_{R}^{\prime}$ is a rational extension of $R_{R}$. Moreover

$$
E\left(R_{R^{\prime}}^{\prime}\right) \text {-domi. } \operatorname{dim} R_{R^{\prime}}^{\prime} \geqq 2,
$$

since $U_{S}$ is a lower distinguished generator for $\mathscr{M}_{S}$ (cf. Morita [14, Theorem 8.2]). Thus $R^{\prime}=Q$ (cf. Tachikawa [18, Corollary 2]), and so ${ }_{Q} U$ is dominant.

REMARK. $Q$ has a dominant left module if and only if, $\mathscr{L}\left(E\left(R_{R}\right)\right)$, the full subcategory of $\mathscr{M}_{R}$ consisting of all modules having $E\left(R_{R}\right)$-dominant dimension $\geqq 2$, is equivalent to $\mathscr{M}_{S}$ for a ring $S$ by Kato [10, Corollary 2] (cf. Morita [14], Tachikawa [17, 18], and Kato [7, 9]).

EXAMPLE 9. Let $R=Z$ be the ring of integers and $Q$ the rational number field. Then there exists an equivalence

$$
\mathscr{L}\left(E\left(Z_{Z}\right)\right)=\mathscr{L}\left(Q_{Z}\right) \sim \mathscr{M}_{Q} \text {. }
$$

LEMMA 4. Let $R$ be a ring and $Q$ Utumi-Lambek maximal right quotient ring of $R$. Suppose $Q$ has a dominant module ${ }_{Q} U$. Then

(1) $T \otimes{ }_{R} U=0 \Leftrightarrow \operatorname{Hom}\left(T_{R}, E\left(R_{R}\right)\right)=0$ for $T_{R} \in \mathscr{M}_{R}$.

(2) $\operatorname{Hom}\left(\operatorname{Hom}\left({ }_{R} Y, Q / R\right)_{R}, E\left(R_{R}\right)\right)=0$ for finitely generated ${ }_{R} Y \in_{R} \mathscr{M}$.

(3) $\operatorname{Hom}\left({ }_{R} Y,{ }_{R} Q\right) \otimes{ }_{R} U \approx \operatorname{Hom}\left({ }_{R} Y,{ }_{R} U\right)$ canonically for ${ }_{R} Y \in \in_{R} \mathscr{M l}$.

(3') $\operatorname{Ext}^{1}\left({ }_{R} X,{ }_{R} Q\right) \otimes{ }_{R} U \approx \operatorname{Ext}^{1}\left({ }_{R} X,{ }_{R} U\right)$ for ${ }_{R} X \in_{R} \mathscr{M}$.

(4) The canonical map

$$
\alpha: \operatorname{Hom}\left({ }_{R} Y,{ }_{R} R\right) \otimes{ }_{R} U \longrightarrow \operatorname{Hom}\left({ }_{R} Y,{ }_{R} U\right)
$$

is a monomorphism (resp. an isomorphism) for ${ }_{R} Y \in_{R} \mathscr{M}$ (resp. for finitely generated $\left.{ }_{R} Y \in_{R} \mathscr{M}\right)$.

(4') There exists a monomorphism (resp. an epimorphism)

$$
\operatorname{Ext}^{1}\left({ }_{R} X,{ }_{R} R\right) \otimes{ }_{R} U \longrightarrow \operatorname{Ext}^{1}\left({ }_{R} X,{ }_{R} U\right)
$$

for finitely generated ${ }_{R} X \in_{R} \mathscr{C l}$ (resp. for finitely related $\left.{ }^{10)}{ }_{R} X \in_{R} \mathscr{C l}\right)$.

Proof. Let $S=\operatorname{End}\left({ }_{Q} U\right)$. Then $Q=\operatorname{End}\left(U_{S}\right)$ and $S=\operatorname{End}\left({ }_{R} U\right)$ as in the above proof.

(1) follows from the isomorphisms

$\operatorname{Hom}\left(T \otimes{ }_{R} U_{S}, E\left(U_{S}\right)\right) \approx \operatorname{Hom}\left(T_{R}, \operatorname{Hom}\left(U_{S}, E\left(U_{S}\right)\right)_{R}\right) \approx \operatorname{Hom}\left(T_{R}, E\left(R_{R}\right)\right)$

and from the fact that $E\left(U_{S}\right)$ is a cogenerator for $\mathscr{M}_{S}$.

(2)

$$
\left.\operatorname{Hom}\left({ }_{R} Y, Q / R\right) \otimes_{R} U G \operatorname{Hom}_{R} Y, Q / R \otimes_{R} U\right)=0,
$$

10) ${ }_{R} X$ is called finitely related if there exists an exact sequence $0 \rightarrow{ }_{R} Y \rightarrow{ }_{R} P \rightarrow{ }_{R} X \rightarrow 0$ with ${ }_{R} P$ projective (not necessarily finitely generated) and ${ }_{R} Y$ finitely generated. 
for, ${ }_{R} Y$ is finitely generated and ${ }_{R} U$ is flat by Theorem 2. It follows that $\operatorname{Hom}\left({ }_{R} Y, Q / R\right) \otimes_{R} U=0$, or equivalently,

$$
\operatorname{Hom}\left(\operatorname{Hom}\left({ }_{R} Y, Q / R\right)_{R}, E\left(R_{R}\right)\right)=0
$$

in view of (1).

(3)

$$
\begin{aligned}
& \operatorname{Hom}\left({ }_{R} Y,{ }_{R} Q\right) \otimes{ }_{R} U_{S} \approx \operatorname{Hom}\left({ }_{R} Y,{ }_{R} \operatorname{Hom}\left(U_{S}, U_{S}\right)\right) \otimes{ }_{R} U_{S} \\
& \approx \operatorname{Hom}\left(U_{S}, \operatorname{Hom}\left({ }_{R} Y,{ }_{R} U\right)_{S}\right) \otimes_{R} U_{S} \approx \operatorname{Hom}\left({ }_{R} Y,{ }_{R} U\right)_{S}
\end{aligned}
$$

canonically for ${ }_{R} Y \in \mathscr{M}$, since ${ }_{R} U$ is of type $F P$ by Theorem 2 (cf. Morita [14, Theorem 1.1]).

(3') An exact sequence $0 \rightarrow{ }_{R} Y \rightarrow{ }_{R} P \rightarrow{ }_{R} X \rightarrow 0$ with ${ }_{R} P$ projective yields an exact commutative diagram

$$
\begin{gathered}
\operatorname{Hom}\left({ }_{R} P,{ }_{R} Q\right) \otimes{ }_{R} U \longrightarrow \operatorname{Hom}\left({ }_{R} Y,{ }_{R} Q\right) \otimes{ }_{R} U \longrightarrow \operatorname{Ext}^{1}\left({ }_{R} X,{ }_{R} Q\right) \otimes{ }_{R} U \longrightarrow 0 \\
\text { U } \\
\left.\operatorname{Hom}\left({ }_{R} P,{ }_{R} U\right) \longrightarrow \operatorname{Hom}\left({ }_{R} Y,{ }_{R} U\right) \longrightarrow \operatorname{Ext}^{1}{ }_{R} X,{ }_{R} U\right) \longrightarrow 0
\end{gathered}
$$

with vertical maps isomorphisms by (3). Thus

$$
\operatorname{Ext}^{1}\left({ }_{R} X,{ }_{R} Q\right) \otimes{ }_{R} U \approx \operatorname{Ext}^{1}\left({ }_{R} X,{ }_{R} U\right) \text { for }{ }_{R} X \in \in_{R} \mathscr{L} \text {. }
$$

(4) Since ${ }_{R} U$ is flat, the exact sequence $0 \rightarrow R \rightarrow Q \rightarrow Q / R \rightarrow 0$ induces the exact commutative diagram for ${ }_{R} Y \in_{R} \mathscr{M}$

$0 \longrightarrow \operatorname{Hom}\left({ }_{R} Y,{ }_{R} R\right) \otimes{ }_{R} U \longrightarrow \operatorname{Hom}\left({ }_{R} Y,{ }_{R} Q\right) \otimes{ }_{R} U \longrightarrow \operatorname{Hom}\left({ }_{R} Y, Q / R\right) \otimes_{R} U$

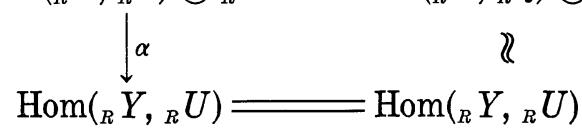

making use of (3). Hence $\alpha$ is a monomorphism for ${ }_{R} Y \in_{R} \mathscr{M}$ and an isomorphism for finitely generated ${ }_{R} Y \in_{R} \mathscr{M}$ by (1) and (2).

$\left(4^{\prime}\right)$ In the situation of $\left(3^{\prime}\right)$, consider the exact commutative diagram

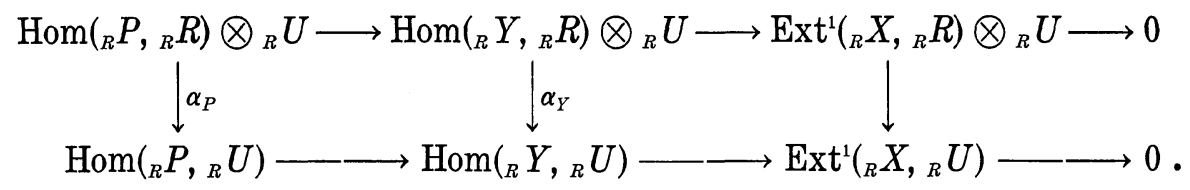

Each of the $\alpha$ 's is a monomorphism and $\alpha_{P}$ (resp. $\alpha_{Y}$ ) is an isomorphism if ${ }_{R} P\left(\operatorname{resp}{ }_{R} Y\right)$ is finitely generated by $(4)$. Thus $\left(4^{\prime}\right)$ follows from Five lemma.

REMARK. The statement (2) in Lemma 4 is still true without the assumption that $Q$ has a dominant left module.

THEOREM 3. Let $R$ be a ring and $Q$ Utumi-Lambek maximal right 
quotient ring of $R$. Assume $Q$ has a dominant left module. Consider now the following conditions:

(1) If ${ }_{Q} U$ is dominant, then ${ }_{R} U$ is injective.

(1') There exists a dominant module ${ }_{Q} U$ such that ${ }_{R} U$ is injective.

(2) $\operatorname{Hom}\left(\operatorname{Ext}^{1}\left({ }_{R} X,{ }_{R} Q\right)_{R}, E\left(R_{R}\right)\right)=0$ for ${ }_{R} X \in_{R} \mathscr{M}$.

(2') $\operatorname{Hom}\left(\operatorname{Ext}^{1}\left({ }_{R} X,{ }_{R} Q\right)_{R}, E\left(R_{R}\right)\right)=0$ for finitely generated ${ }_{R} X \in_{R} \mathscr{L}$.

$\left.\left(2^{\prime \prime}\right) \operatorname{Hom}\left(\operatorname{Ext}^{1}{ }_{R} X,{ }_{R} R\right)_{R}, E\left(R_{R}\right)\right)=0$ for finitely generated ${ }_{R} X \in_{R} \mathscr{M}$.

$\left(1^{\prime \prime}\right)$ If ${ }_{Q} U$ is dominant, then $\operatorname{Ext}^{1}\left({ }_{R} X,{ }_{R} U\right)=0$ for finitely presented ${ }_{R} X \in_{R} \mathscr{M}$.

(3) $E\left({ }_{R} R\right)$ is flat.

Then $(1) \Leftrightarrow\left(1^{\prime}\right) \Leftrightarrow(2) \Leftrightarrow\left(2^{\prime}\right) \Rightarrow\left(2^{\prime \prime}\right) \Rightarrow\left(1^{\prime \prime}\right)$, and if $R$ is left Noetherian they all are equivalent.

Proof. $(1) \Leftrightarrow\left(1^{\prime}\right) \Leftrightarrow(2) \Leftrightarrow\left(2^{\prime}\right) \Rightarrow\left(2^{\prime \prime}\right) \Rightarrow\left(1^{\prime \prime}\right)$ by Lemma 4 .

From now on, suppose $R$ is left Noetherian. Then

$\left(1^{\prime \prime}\right) \Rightarrow(1)$ is well-known.

$\left(1^{\prime}\right) \Rightarrow(3)$. Since ${ }_{R} U$ is faithful and injective,

$$
E\left({ }_{R} R\right) \subset \Pi_{R} U \text {. }
$$

Hence $E\left({ }_{R} R\right)$ is flat by Theorem 2 and Cartan and Eilenberg [3, Exercise 4, p. 122].

$(3) \Rightarrow\left(2^{\prime \prime}\right)$ is due to Morita [15, Theorem 2].

\section{REFERENCES}

[1] G. Azumaya, Completely faithful modules and self-injective rings, Nagoya Math. J., 27 (1966), 697-708.

[2] H. BASS, The Morita theorems, University of Oregon, Lecture notes 1962.

[3] H. Cartan and S. Eilenberg, Homological algebra, Princeton, University Press 1956.

[4] R. R. Colby and E. A. RUtter, Jr., Semi-primary QF-3 rings, Nagoya Math. J., 32 (1968), 253-258.

[5] J. P. Jans, Duality in Noetherian rings, Proc. Amer. Math. Soc., 12 (1961), 829-835.

[6] T. Kato, Torsionless modules, Tôhoku Math. J., 20 (1968), 234-243.

[ 7$]$ T. Kato, Rings of dominant dimension $\geqq 1$, Proc. Japan Acad., 44 (1968), 579-584.

[8] T. KATo, Rings of $U$-dominant dimension $\geqq 1$, Tôhoku Math. J., 21 (1969), 321-327.

[9] T. Kato, Dominant modules, J. Algebra, 14 (1970), 341-349.

[10] T. KАто, $U$-dominant dimension and $U$-localization, Unpublished.

[11] J. LAMBeK, On Utumi's ring of quotients, Canad. J. Math., 15 (1963), 363-370.

[12] K. Morita, Adjoint pairs of functors and Frobenius extensions, Sci. Rep. Tokyo Kyoiku Daigaku, Sect. A9, No. 205 (1965), 40-71.

[13] K. MoRITA, Duality in QF-3 rings, Math. Z., 108 (1969), 237-252.

[14] K. Morita, Localizations in categories of modules, Math. Z., 114 (1970), 121-144.

[15] K. Morita, Noetherian QF-3 rings and two-sided quasi-Frobenius maximal quotient rings, Proc. Japan Acad., 46 (1970), 837-840.

[16] T. ONODERA, Koendlich erzeugte Moduln und Kogeneratoren, To appear.

[17] H. Tachikawa, On left QF-3 rings, Pacific J. Math., 32 (1970), 255-268. 
[18] H. TaChikawa, On splitting of module categories, Math. Z., 111 (1969), 145-150.

[19] U. Utumi, On quotient rings, Osaka Math. J., 8(1956), 1-18.

DEPARTMENT OF MATHEMATICS

College of General Education

TôHOKU UNIVERSITY

KaWAUChI, SendaI, JAPAN 